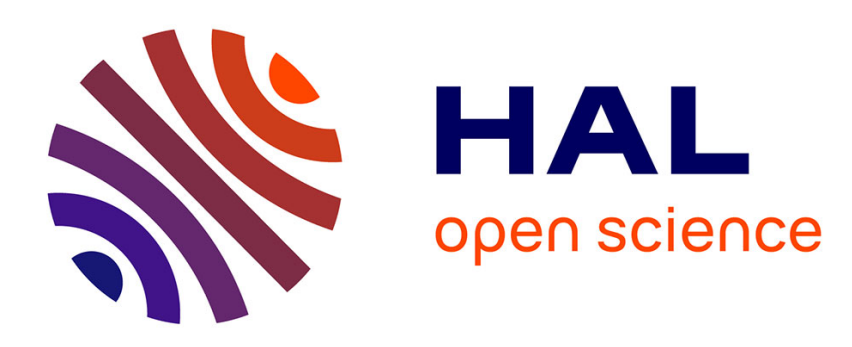

\title{
The distribution of EM waves scattering from cutaneous surface
}

\author{
T. Galal, D. Rondot, Jean Mignot
}

\section{To cite this version:}

T. Galal, D. Rondot, Jean Mignot. The distribution of EM waves scattering from cutaneous surface. Revue de Physique Appliquée, 1989, 24 (4), pp.469-476. 10.1051/rphysap:01989002404046900 . jpa00246069

\section{HAL Id: jpa-00246069 https://hal.science/jpa-00246069}

Submitted on 1 Jan 1989

HAL is a multi-disciplinary open access archive for the deposit and dissemination of scientific research documents, whether they are published or not. The documents may come from teaching and research institutions in France or abroad, or from public or private research centers.
L'archive ouverte pluridisciplinaire HAL, est destinée au dépôt et à la diffusion de documents scientifiques de niveau recherche, publiés ou non, émanant des établissements d'enseignement et de recherche français ou étrangers, des laboratoires publics ou privés. 


\title{
The distribution of EM waves scattering from cutaneous surface
}

\author{
T. Galal ( $\left.{ }^{1}\right)$, D. Rondot $\left({ }^{1, *}\right)$ and J. Mignot $\left(^{(1)}\right.$ \\ (1) Laboratoire de Biologie Cutanée, Faculté de Médecine, 25030 Besançon, France
}

(Reçu le 16 octobre 1987, révisé le 8 décembre 1988, accepté le 16 décembre 1988)

\begin{abstract}
Résumé. - La solution discrétisée générale et explicite du modèle mathématique à deux dimensions de Beckmann, relatif à la diffusion des ondes électromagnétiques par une interface rugueuse (surface cutanée), a été calculée numériquement et trouvée en accord avec les paramètres topographiques quantitatifs obtenus de cette surface à l'aide d'un profilomètre automatique à large échelle.
\end{abstract}

\begin{abstract}
The general explicit discretized solution for the Beckmann mathematical 2-D model, for the scattering of electromagnetic waves, from a rough boundary (a cutaneous surface was selected for application) was determined numerically and found in accordance with the reality of its topographical quantitative parameters, obtained by a 3-D automated wide range profilometer.
\end{abstract}

\section{Introduction.}

When an electromagnetic wave is incident on an irregular boundary with random variations of heights, where roughness does not include sharp edges or sharp points, the reflected field mainly depends on the wave length, the angle of incidence, the surface geometry, and the electrical properties (permittivity, permeability, and conductivity) of both media.

In attemps to predict the distribution of EM waves scattered from rough surfaces, several models have been proposed. One of the most interesting mathematical models, up to now, is the Beckmann model. However, it is known that, if the conductivity of the surface is finite, no solution being general, exact and explicit at the same time, is at present known.

We have determined the Beckmann general and explicit solution numerically, in two dimensions. A special apparatus was used to obtain the necessary amount of information, as could be detected from surface geometry.

(*) Institut Universitaire de Technologie, rue EngelGros, 90016 Belfort, France.

\section{Approximate solution.}

According to Beckmann [1], the exact and general solution for the field scattered from a rough surface with finite conductivity in two dimensions is given by (see appendix A) :

$$
\begin{array}{r}
\rho=\frac{1}{8 X Y \cos \theta_{1}} \int_{-x}^{x} \int_{-y}^{y}\left(a \zeta_{x}^{\prime}+c \zeta_{y}^{\prime}-b\right) \times \\
\times \mathrm{e}^{i \mathbf{v} \cdot \mathbf{r}} \mathrm{d} x \mathrm{~d} y
\end{array}
$$

where

$$
\begin{gathered}
\mathbf{V}=k\left[\left(\operatorname{Sin} \theta_{1}-\operatorname{Sin} \theta_{2} \operatorname{Cos} \theta_{3}\right) \mathbf{X}_{0}-\right. \\
\left.-\operatorname{Sin} \theta_{2} \operatorname{Sin} \theta_{3} \mathbf{Y}_{0}-\left(\operatorname{Cos} \theta_{1}+\operatorname{Cos} \theta_{2}\right) \mathbf{Z}_{0}\right], \\
k=2 \pi / \lambda, \\
\mathbf{r}=x \mathbf{X}_{0}+y \mathbf{Y}_{0}+\zeta(x, y) \mathbf{Z}_{0}, \\
a=(1-R) \operatorname{Sin} \theta_{1}+(1+R) \operatorname{Sin} \theta_{2} \operatorname{Cos} \theta_{3}, \\
b=(1+R) \operatorname{Cos} \theta_{2}-(1-R) \operatorname{Cos} \theta_{1}, \\
c=(1+R) \operatorname{Sin} \theta_{2} \operatorname{Sin} \theta_{3}, \\
R=\frac{\Lambda^{2} \operatorname{Cos} \nu-\sqrt{\left(\Lambda^{2}-\operatorname{Sin}^{2} \nu\right)}}{\Lambda^{2} \operatorname{Cos} \nu+\sqrt{\left(\Lambda^{2}-\operatorname{Sin}^{2} \nu\right)}} \\
\nu=\theta_{1}-\arctan \zeta_{x}^{\prime}, \\
\Lambda=\sqrt{\frac{\varepsilon_{\mathrm{rc}}}{\mu_{\mathrm{rc}}}}
\end{gathered}
$$


and

$$
\varepsilon_{\mathrm{rc}}=\frac{\varepsilon}{\varepsilon_{0}}-i 60 \lambda \Omega
$$

It was assumed that the surface is isotropically rough, i.e. that $\zeta$ is distributed with the same statistical distribution $W(Z)$, in all directions over the surface. In the contrary case, when the distribution is different for the $X$ and $Y$ directions, the calculation becomes very complicated and therefore the solution for the general anisotropic case was not discussed [1]. However in practical applications, anisotropic roughness occurs for skin surfaces having a different distribution along different scanning directions ; in this case Beckmann [1] suggested the use of only one dimensional roughness, in a way of approximation (which is but a simple special case of anisotropic roughness) regarding the surface of the skin as constant in other directions, such as :

$$
W(Z)=\frac{1}{\sigma \sqrt{(2 \pi)}} \mathrm{e}^{-Z^{2} / 2 \sigma^{2}} .
$$

For a random rough surface the reflection coefficient $R$ is a random function of $\zeta^{\prime}$, since the local angle of incidence $\nu$ is a function of $\zeta^{\prime}$. In a stationary random process $\zeta$ and $\zeta^{\prime}$ are everywhere uncorrelated. Assuming as in [1] $\langle\zeta\rangle=0$ and $\left\langle\zeta^{\prime}\right\rangle=0$

$$
\left\langle R\left(\zeta^{\prime}\right)\right\rangle=R\left(\nu=\theta_{1}\right) .
$$

Beckmann calls the polarization of $\mathbf{E}_{1}$, vertical if $\mathbf{E}_{1}$ lies in the plane of incidence $\mathbf{K}_{1}, \mathbf{Z}_{0}$ and $\mathbf{E}_{2}$ is called vertically polarized if it lies in the scattering plane $\mathbf{K}_{2}, \mathbf{Z}_{0}$. Similarly, in horizontal polarization $\mathbf{E}_{1}$ and $\mathbf{E}_{2}$ are normal to the plane of incidence and the scattering plane respectively, while one ignores the question of whether the polarization of the scattered wave is the same as that of the incident wave or in the contrary case, « depolarization » sets in [1].

The average scattered field for finite conductivity : $\langle\rho\rangle_{\mathrm{f}}$ is approximated by :

$\langle\rho\rangle_{\mathrm{f}}=\langle R\rangle\langle\rho\rangle_{\infty}$ as in reference [1] (Sect. 5.4)

where the subscripts $f$ and $\infty$ denote finite and infinite conductivity respectively and $\langle R\rangle$ is given approximately by (13).

But for infinite conductivity the average field is :

$$
\langle\rho\rangle_{\infty}=F_{3} \cdot \operatorname{sinc} v_{x} X \cdot \operatorname{sinc} v_{y} Y \cdot \mathrm{e}^{-\sigma^{2} v_{z}^{2} / 2}
$$

with

$$
F_{3}=\frac{1+\operatorname{Cos} \theta_{1} \operatorname{Cos} \theta_{2}-\operatorname{Sin} \theta_{1} \operatorname{Sin} \theta_{2} \operatorname{Cos} \theta_{3}}{\operatorname{Cos} \theta_{1}\left(\operatorname{Cos} \theta_{1}+\operatorname{Cos} \theta_{2}\right)},
$$

$\operatorname{sinc} v_{x} X=\frac{\sin v_{x} X}{v_{x} X} \quad$ and $\quad \operatorname{sinc} v_{y} Y=\frac{\sin v_{y} Y}{v_{y} Y}$

The value of skin admittance, at a given frequency is not considered here, because it is not at hand, and because the admittance of the media has a direct impact on the reflected field levels, but not on the field distribution over the surface, which is the matter of concern in this paper. Also it is wellknown that the surface geometry modifies the scattered field far more than its electrical properties; this is also borne out by experimental measurements [1].

For the sake of a 3-D representation : if $\sigma / \lambda \gg$ $10^{-4}$, this model will not be suitable, due to the rapid change of the sinc term, while the exponential term has a constant value for $10^{-2}>\sigma / \lambda>10^{-4}$. So, we chose $\sigma / \lambda$ within a $10^{-4}$ range to slow down the variation of the sinc term [3].

Figure 1 represents the skin surface under consideration, while figure 2 gives the ISO contour

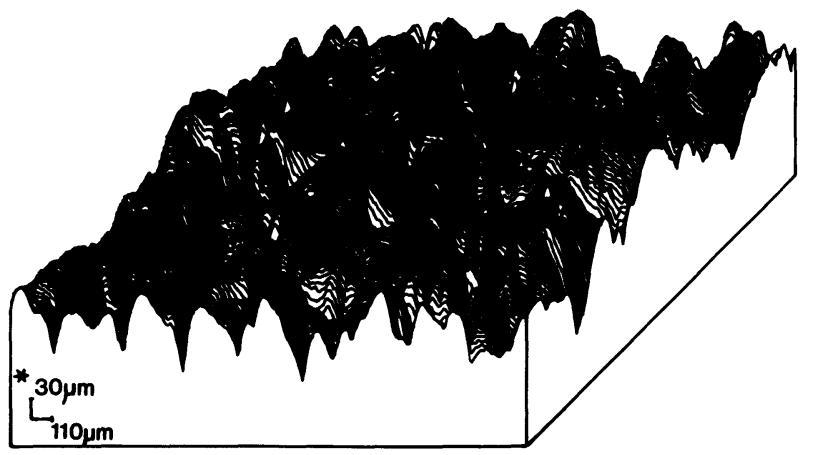

Fig. 1. - Skin surface under consideration. $\left({ }^{*}\right)$ The used unit scale in $(x, y)$ and $z$ directions.

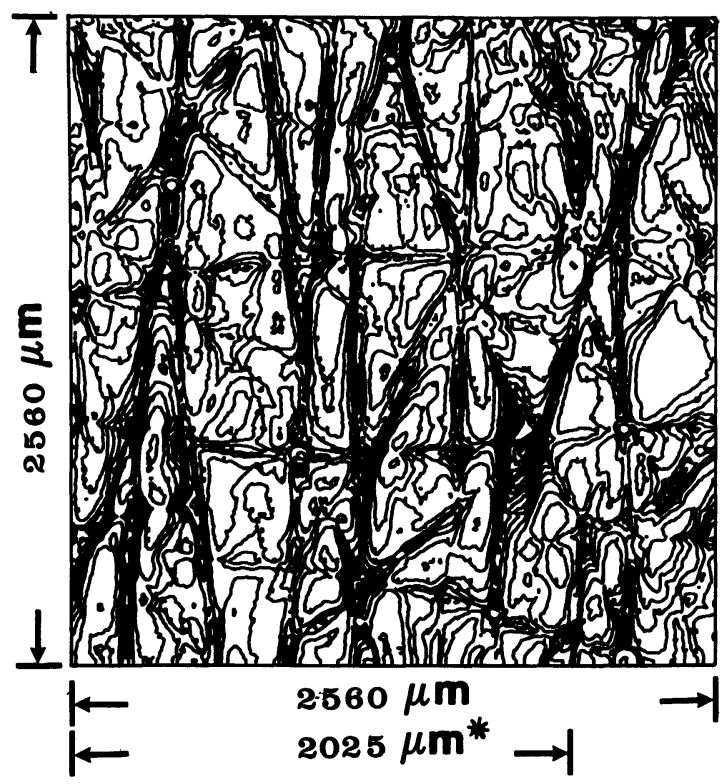

Fig. 2. - ISO contour levels of the given cutaneous relief (surface extension in : $\pm X_{0}$ direction $=2560 \mu \mathrm{m}$, and in $\pm Y_{0}$ direction $\left.=2560 \mu \mathrm{m}\right)$. $\left({ }^{*}\right)$ See figure 10 . 
levels map for this surface, and figure 3 is the distribution of $\langle\rho\rangle_{f}$ as a function of $\theta_{1}$ and $\theta_{2}$.

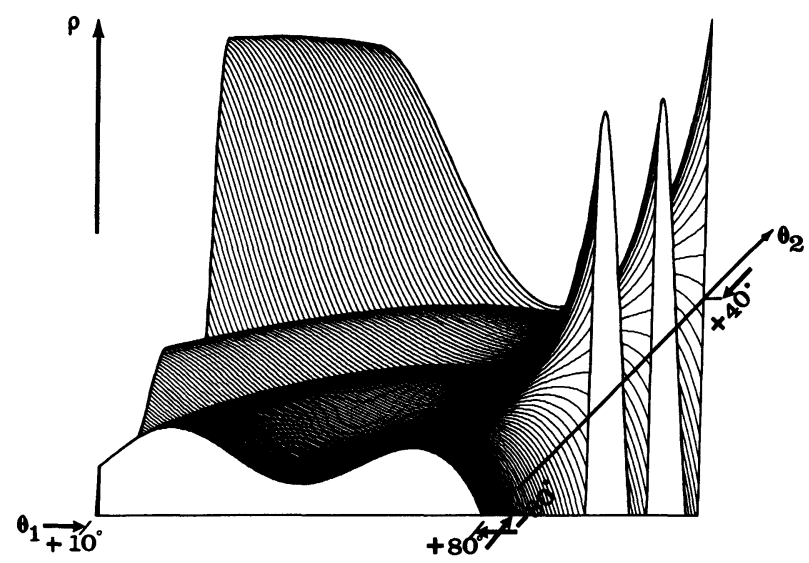

Fig. 3. - Distribution of $\langle\rho\rangle_{\mathrm{f}}$ as a function of $\theta_{1}$ (in the plane of the paper), and $\theta_{2}$ ( $\perp$ to the plane of the paper), $\theta_{3}=0, \Lambda=2$, and $\sigma / \lambda=5 \times 10^{-4}$ with $\sigma=50 \mu \mathrm{m}$, $\rho_{\text {max }}=0.376$, and $\rho_{\text {min }}=-0.092$. $\theta_{1}$ varies from $+10^{\circ}$ to $+80^{\circ}$ and $\theta_{2}$ varies from $-80^{\circ} \rightarrow+40^{\circ}$; a reduced span of $\theta_{2}$ is used (usually the same max as for $\theta_{1}$ is considered) to simplify the figure.

\section{The general and explicit two dimensional numeri-} cal discretization solution.

We discretized the considered surface of dimensions $2560 \mu \mathrm{m} \times 2560 \mu \mathrm{m}$ (see Fig. 4), into elements, each of the dimensions $20 \mu \mathrm{m} \times 20 \mu \mathrm{m}$, using a three dimensional automated wide range profilometer to determine $\zeta_{x}, \zeta_{x}^{\prime}, \zeta_{y}, \zeta_{y}^{\prime}, \bar{\zeta}$ and $\sigma$ (see Fig. 5) for each element, at the points of measurement indicated in figure 4 where they have certain values for its associated element and where the measurement step is $\Delta X=20 \mu \mathrm{m}$ in $X$ direction and
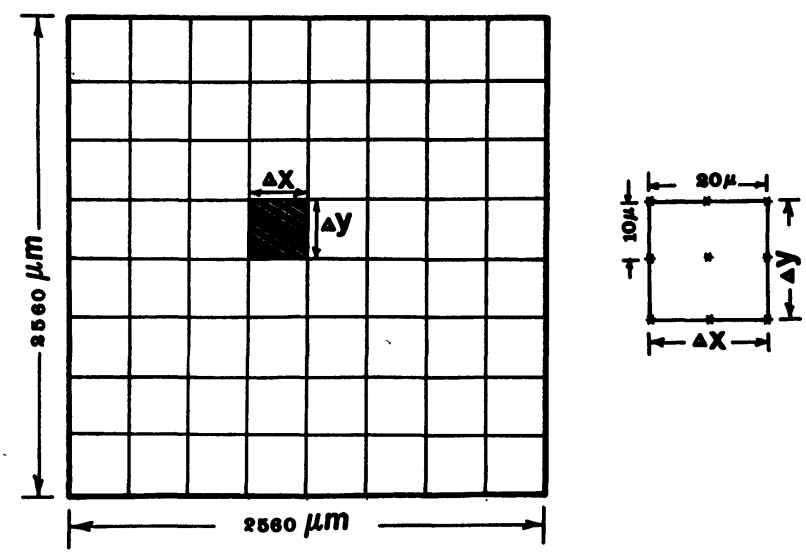

Fig. 4. - Discretized surface with $\Delta x=20 \mu \mathrm{m}$ and $\Delta y=20 \mu \mathrm{m}$, identifying the measurement points locally on an element's surface, and boundaries by asterisks.

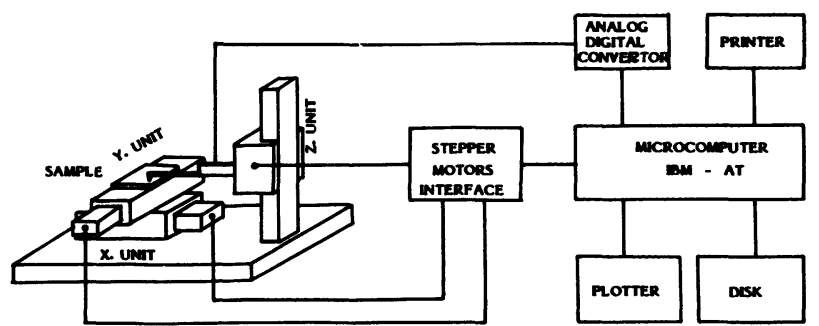

Fig. 5. - Block diagram of the 3-D profilometer's measurement facility.

$\Delta Y=20 \mu \mathrm{m}$ in $Y$ direction. We assume that over the element certain mathematical probability distributions are known for $\zeta_{x}$ and $\zeta_{y}$ involving that the elementary surface is isotropically rough, i.e. $\zeta$ is distributed with the same statistical distribution $W(Z)$ in all directions $(X, Y)$, see figure 6 . There is practically no difference if a surface is rough in only one dimension or isotropically rough in two dimensions.

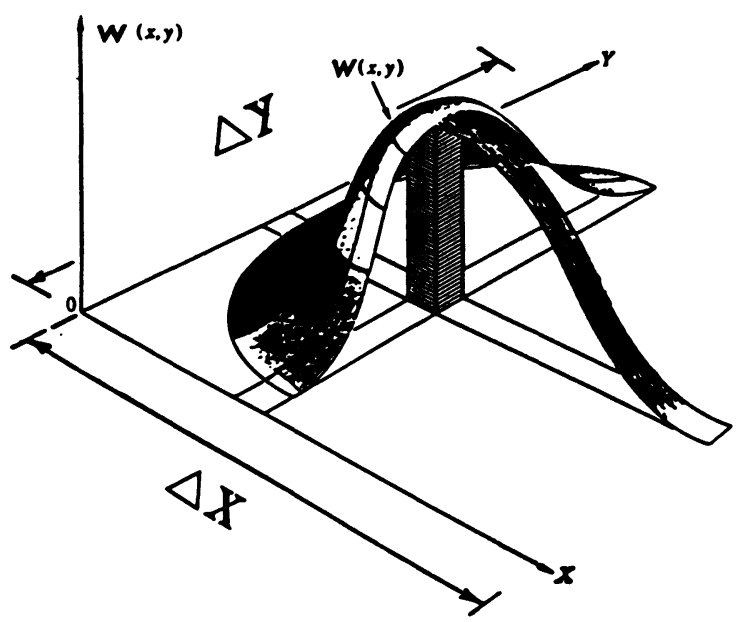

Fig. 6. - A two dimensional probability density of local heights $W(z): \zeta$ shows the same statistical distribution in all directions.

We have chosen the incident wave to be vertically polarized (see appendix B, for the definition of the reference field $E_{20}$ ), while we ignore the polarization state of the scattered wave, for it is not in the field of interest of this paper.

$E_{2}$ over each element is given by :

$E_{2}^{(\mathrm{e})}=\frac{i k \mathrm{e}^{i k R_{0}}}{4 \pi R_{0}} \int_{-x}^{x} \int_{-y}^{y}\left(a \zeta_{x}^{\prime}+c \zeta_{y}^{\prime}-b\right) \mathrm{e}^{i \mathrm{v} \cdot \mathrm{r}} \mathrm{d} x \mathrm{~d} y$.

The average scattering coefficient from one element $\langle\rho\rangle^{(\mathrm{e})}$ is given by :

$$
\langle\rho\rangle^{(\mathrm{e})}=\left\langle\frac{E_{2}}{E_{20}}\right\rangle
$$


with $E_{20}$ given by equation (B.6) or $\langle\rho\rangle^{(\mathrm{e})}$ would be written as :

$$
\begin{aligned}
\langle\rho\rangle^{(\mathrm{e})} & =\frac{1}{-8 X Y R \cos \theta_{1}} \times \\
& \times \int_{-x}^{x} \int_{-y}^{y}\left(a \zeta_{x}^{\prime}+c \zeta_{y}^{\prime}-b\right) \mathrm{e}^{i \mathbf{v} \cdot \mathbf{r}} \mathrm{d} x \mathrm{~d} y .
\end{aligned}
$$

After evaluating the integration of equation (20) over the elementary surface $\Delta X \times \Delta Y$, and by neglecting any interaction between adjacent elements, including the edge effect which will be shown negligible when $\Delta X \times \Delta Y \gg \lambda^{2}$ and assuming a Gaussian distribution of the random parameter in $Z$ direction over each surface element, with certain mean value $\bar{\zeta}$, and a standard deviation $\sigma$, the elemental average scattering coefficient in its final form $\langle\rho\rangle^{(e)}$ is given by :

$$
\begin{aligned}
\langle\rho\rangle^{(\mathrm{e})}=\frac{a v_{x}+b v_{z}+c v_{y}}{-2 R v_{z} \cos \theta_{1}} \operatorname{sinc} v_{x} X \times \\
\quad \times \operatorname{sinc} v_{y} Y \cdot \cos \left(v_{z} \bar{\zeta}\right) \cdot \mathrm{e}^{-\sigma^{2} v^{2} / 2}
\end{aligned}
$$

with $a, b$ and $c$ given by equations ((5), (6) and (7)) respectively.

We have examined equation (21), to ensure that it satisfies the case of grazing incidence, where we find

when

$$
\begin{gathered}
\theta_{1} \rightarrow \pi / 2 \text { and } \theta_{2} \rightarrow \pi / 2 \\
\langle\rho\rangle^{(\mathrm{e})} \rightarrow 1
\end{gathered}
$$

now we are certain this model confirms the associated imposed condition.

As has been shown above, we evaluated the average value of the scattered field over one element ; $\Delta X \Delta Y=20 \mu \mathrm{m} \times 20$, represented by a point $« \mathrm{p} »$ on the scattering pattern side (see Fig. 7).

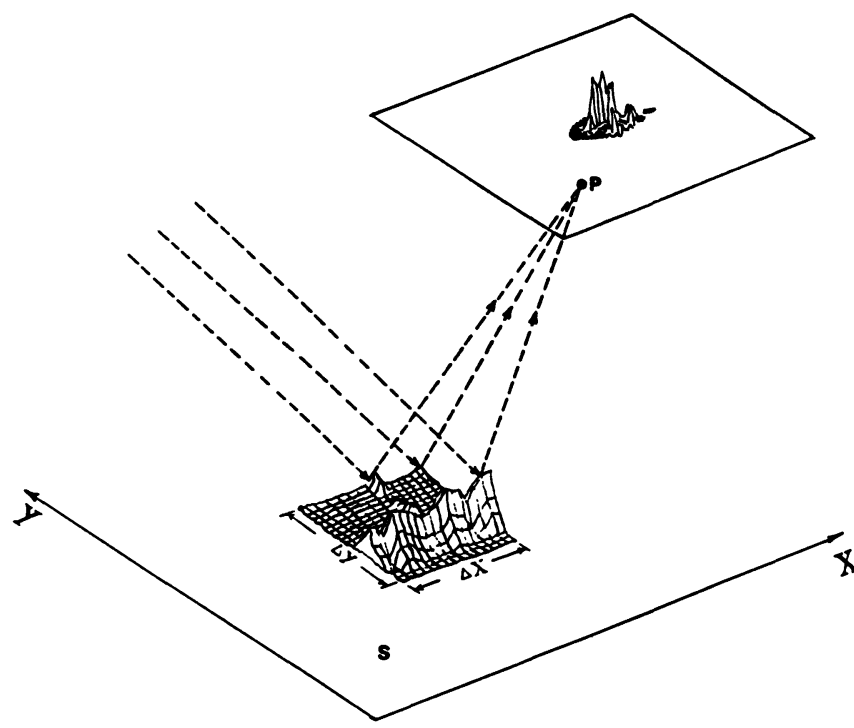

Fig. 7. - Describes the reflection of each element $\Delta x \Delta y$ on the surface side to one point $« \mathrm{p} »$ on the EM Waves scattering pattern side.

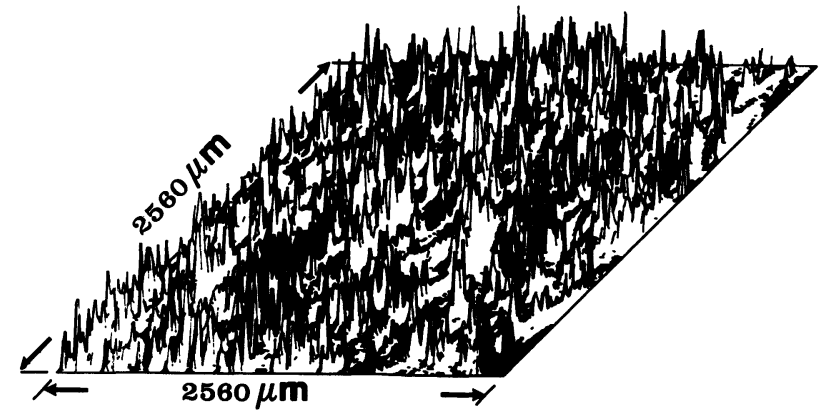

Fig. 8. - 2-D distribution of the scattering coefficient $\rho$ over a given cutaneous relief $(\lambda=5 \mu \mathrm{m}, \Lambda=2$, $\left.\theta_{1}=20^{\circ}, \theta_{2}=40^{\circ}, \theta_{3}=0^{\circ}\right), \rho_{\max }=-8.967 \mathrm{db}$.

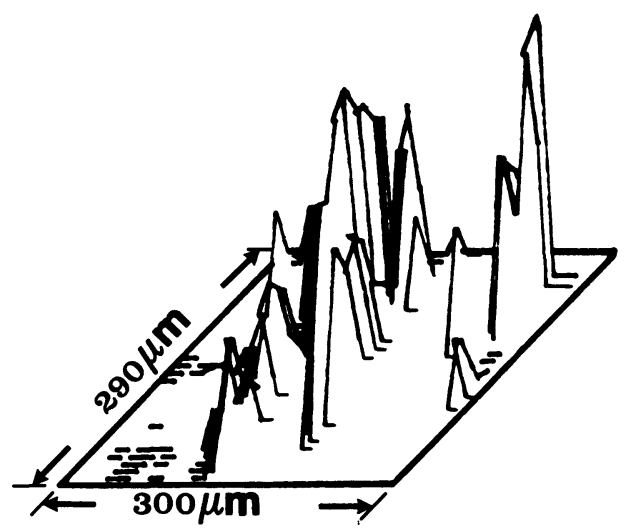

Fig. 9. - Distribution of $|\rho|$ over one of the skin plateaus, with $x=1100 \mu \mathrm{m}, y=10 \mu \mathrm{m}, \Delta x=300 \mu \mathrm{m}$, $\Delta y=290 \mu \mathrm{m} \quad\left(\lambda=5 \mu \mathrm{m}, \quad \Lambda=2, \quad \theta_{1}=20^{\circ}, \quad \theta_{2}=40^{\circ}\right.$, $\left.\theta_{3}=0^{\circ}\right), \quad|\rho|_{\max }=-9.304 \mathrm{db}$.

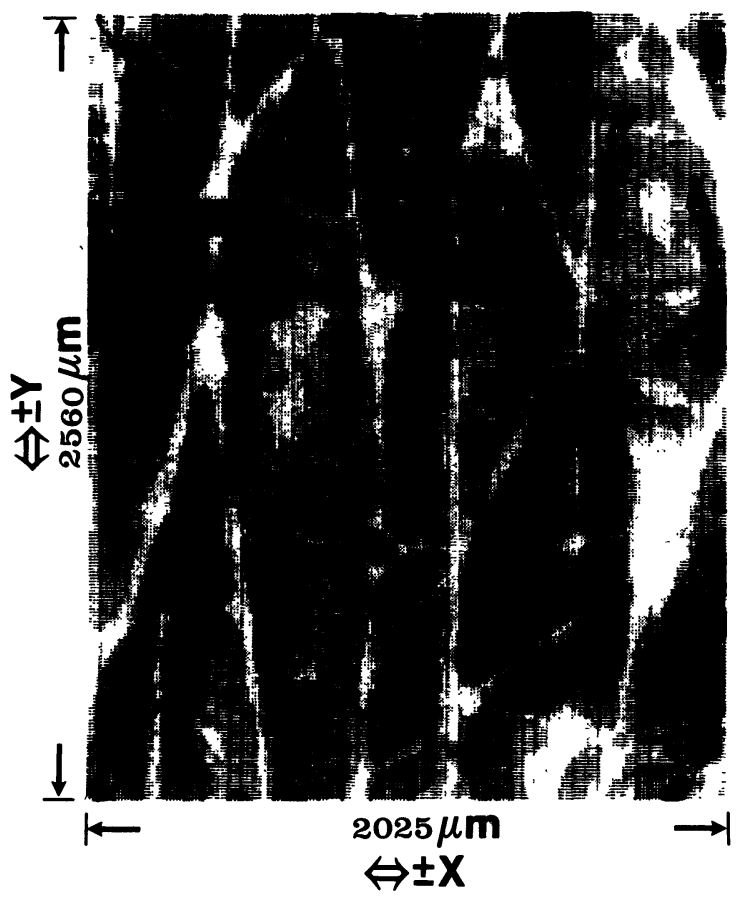

Fig. 10. - ISO level distribution of skin's $\left({ }^{*}\right)$ plateaus and furrows, 7 levels (examples ; white : $0 \mu \mathrm{m}$, black squares : $74.3 \mu \mathrm{m}$, and black (the highest level) : $91.4 \mu \mathrm{m}$ ). (*) The most part of the skin is presented : $2025 \mu \mathrm{m}$ in $\pm X_{0}$ direction and $2560 \mu \mathrm{m}$ in $\pm X_{0}$ direction, see figure 2 . 


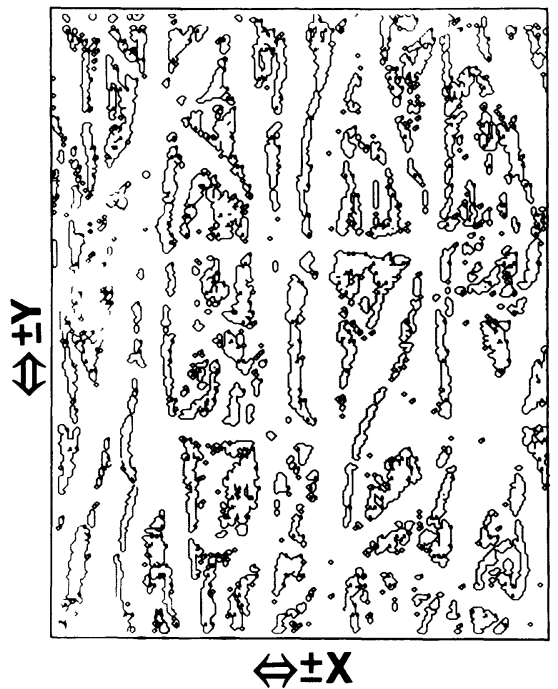

Fig. 11. - Structure of equilevel $\rho$ contours map over skin (given in Fig. 10), at the value $\rho=-14.26 \mathrm{db}$.

While for the surface in the given dimensions : $2560 \mu \mathrm{m} \times 2560 \mu \mathrm{m}$, we expect to have $128 \times$ 128 points over the 2-D scattering pattern side, representing the global variation of the local average scattering coefficient for the surface under consideration.

Figure 8 shows the 2-D distribution of the scattering coefficient $\rho$ over the given cutaneous relief, at $5.996 \times 10^{13} \mathrm{~Hz}, \quad(\lambda=5 \mu \mathrm{m})$ while figure 9 shows the distribution of $|\rho|$ over one of the skin plateaus at the same frequency, and figure 10 shows

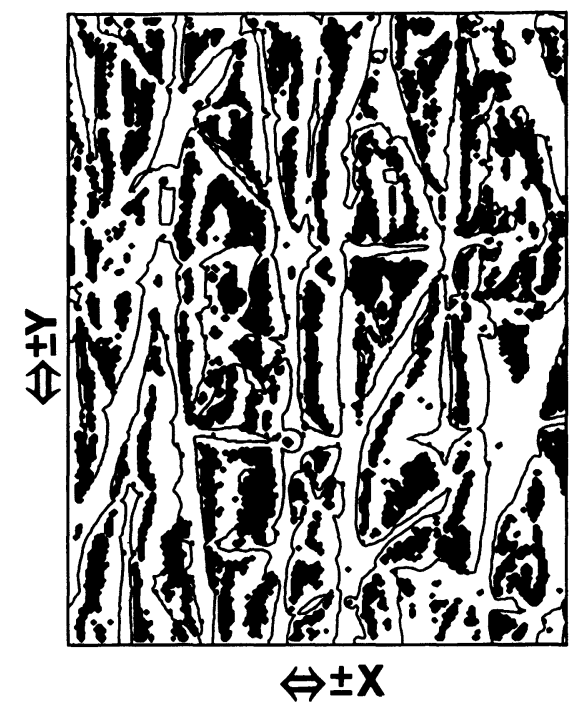

Fig. 12. - Superposition of equilevel $\zeta(x, y)$ contours of skin plateau at the level $73.3 \mu \mathrm{m}$ shown in figure 10 , and equilevel contours at the value $-14.26 \mathrm{db}$ appearing in figure 11 .

the ISO level distribution of $\zeta(x, y)$ on the same skin plateaus and furrows. Figure 11 shows the structure of equilevel contours map of $|\rho|$ over the same surface and figure 12 is a map where the equilevel contours $\zeta$ of the skin (at the highest plateau level) are superimposed on the equilevel contours of $\rho$ over the same area. This figure shows clearly that most of the reflection occurs on the highest plateaus; this coincides with the reality of reflection from irregular boundaries.

\section{A summary for intended outcomes and imposed assumptions of the included solutions.}

Item $\mathrm{N}^{\circ} \quad$ The approximate solution.

$$
<\rho>\text { is determined over the whole surface. }
$$

Assuming a Gaussian distribution over the surface.

$\langle\zeta>=0, \zeta$ is the random parameter in $Z$ direction.

$\left\langle\zeta^{\prime}\right\rangle=0, \zeta^{\prime}$ is the gradient of the random parameter.

$$
<R\left(\zeta^{\prime}\right)>=R\left(\nu=\theta_{1}\right)
$$

\section{Conclusion.}

We performed the Beckmann approximate solution and the general explicit two dimensional numerical discretized solution. From the latter we have ob-
The general and explicit two-dimensional numerical discretization solution.

The distribution of $\rho$ over the surface is determined.

Assuming a Gaussian distribution over the stepping distance $\Delta X \times \Delta Y$ with certain $\bar{\zeta}, \sigma$ (the mean value and the standard deviation respectively). While we could have any distribution over the global surface.

$\zeta$ is measured using 3-D profilometer over each stepping element.

$\zeta^{\prime}$ is determined by making use of the profilometer.

$R\left(\zeta^{\prime}\right)$ varies according to $\zeta^{\prime}$. tained the distribution of the electromagnetic field scattered in accordance with the topographical quantitative parameters of a given surface. The main advantage of this method lays in the capability of the technique to determine the distribution character- 
istics of the field scattered from various elements of a rough surface, assuming a local Gaussian distribution of the random parameter in $Z$ direction over each surface element, with certain mean value, and a standard deviation. The nodal values of the surface heights and its derivatives in $X$, and $Y$ directions have been determined using a 3-D profilometer. While the former method only gives an average (constant) value of the scattered field from the whole surface under consideration, assuming a global Gaussian distribution of the random parameter in $Z$ direction, with a zero mean value, and a given standard deviation.

\section{Nomenclature.}

a : a parameter, is given by equation (5)

$b \quad$ : a parameter, is given by equation (6)

c : a parameter, is given by equation (7)

$F_{3} \quad:$ a parameter, is given by equation (16)

$k \quad:$ the wave number, where $k=\frac{2 \pi}{\lambda}$

$R \quad$ : Fresnel reflection coefficient for a smooth plane

$\mathbf{r} \quad:$ the radius vector

V : vector, is given by $\mathbf{V}=\mathbf{K}_{1}-\mathbf{K}_{2}$, where $K_{1}$ is the propagation vector and $K_{2}$ is the scattered vector

$v \quad$ : the component of vector $\mathbf{V}$

$W \quad:$ the probability height distribution

$x$ and $X_{0}$ : the variable and its Cartesian coordinate

$y$ and $Y_{0}$ : the variable and its Cartesian coordinate

$z$ and $Z_{0}$ : the variable and its Cartesian coordinate

$X \quad$ : the extension of the surface in $(+) X$ direction

$Y \quad$ : the extension of the surface in (+) $Y$ direction

$E_{20} \quad:$ the field reflected in the direction of specular reflection $\left(\theta_{1}=\theta_{2}\right)$ by a smooth, finite conductive plane with the same dimensions, under the same angle of incidence and at the same distance, when the incident wave is vertically polarized.

\section{Greek symbols}

$\begin{array}{ll}\rho & : \text { the scattering coefficient } \\ \langle\rho\rangle(\mathrm{e}) & \begin{array}{l}: \text { the average scattering coefficient for } \\ \text { one element }\end{array} \\ \theta_{1} & \begin{array}{l}: \text { the angle of incidence } \\ \theta_{2}\end{array} \\ & \begin{array}{l}: \text { the scattering angle included in the } \\ \text { plane of incidence }\end{array} \\ \theta_{3} & \begin{array}{l}: \text { an angle introduced for lateral scat- } \\ \text { tering out of the plane of incidence }\end{array} \\ \nu & \begin{array}{l}: \text { local angle of incidence } \\ \text { the surface height variable ; it varies }\end{array} \\ \zeta(x, y) & : \text { the average height over an element }\end{array}$

$$
\begin{array}{ll}
\zeta_{x}^{\prime} & : \frac{\partial \zeta(x, y)}{\partial x} \\
\zeta_{y}^{\prime} & : \frac{\partial \zeta(x, y)}{\partial y} \\
\lambda & : \text { the wave length } \\
\Lambda & : \text { the normalized admittance of the } \\
& \text { medium } \\
\varepsilon_{\mathrm{rc}} & : \text { the relative complex permittivity } \\
\mu_{\mathrm{rc}} & : \text { the relative permeability } \\
\varepsilon & : \text { the dielectric constant of the considered } \\
& \text { medium } \\
\varepsilon_{0} & : \text { the dielectric constant of the free space } \\
\Omega & : \text { the conductivity of the considered } \\
& \text { medium } \\
\sigma & : \text { the standard deviation of the } \zeta \text { variable }
\end{array}
$$

\section{Subscripts}

$$
\begin{array}{ll}
\mathrm{f} & : \text { finite conductivity } \\
\infty & \text { : infinite conductivity. }
\end{array}
$$

\section{Appendix A.}

\section{Corrections applied to some Beckmann equations.}

The following equations given by Beckmann [1] were corrected, either due to typing errors or for other reasons. In most of the latter cases, we give derivations in more details.

We used to express the incident field as :

$$
E_{1}=A \mathrm{e}^{i \phi}
$$

with $A$ the amplitude and $\phi$ the phase.

Here in our case, $E_{1}$ is a harmonic plane wave of unit amplitude, therefore :

$$
A=1 \text { with } \quad \phi=\mathbf{K}_{1} \cdot \mathbf{r}-\omega t
$$

then equation (3) (Sect. 3.1) must be written :

$$
E_{1}=\mathrm{e}^{i\left(\mathbf{K}_{1} \cdot \mathbf{r}-\omega t\right)} .
$$

Spizzichino [1] and beckmann [2] give the exact formula for equation (16) (Sect. 3.1) as follows :

$$
\varepsilon_{\mathrm{rc}}=\frac{\varepsilon}{\varepsilon_{0}}-i 60 \lambda \Omega
$$

Beckmann gives equation (18) (Sect. 3.1) without imposing conditions on $\zeta^{\prime}(x)$, while the incident wave has a known and a definite direction of propagation. But when this model is used, we can see that we must impose a condition on $\zeta^{\prime}(x)$ as indicated below, in order to only obtain the field scattered from that part of the surface facing the incident wave (shadowing effect).

Then equation (18) (Sect. 3.1) should be given as :

$$
\begin{aligned}
& \nu_{x}=\theta_{1}-\beta=\theta_{1}-\arctan \zeta^{\prime}(x) \\
& \text { with } \zeta^{\prime}(x) \geqslant 0 .
\end{aligned}
$$


From equation (31) (Sect. 3.1) we realize that :

$$
v_{x}=k\left(\sin \theta_{1}-\sin \theta_{2}\right) \text {. }
$$

Therefore equation (21) (Sect. 3.1) expressing the vector $\mathbf{V}$ as follows :

$\mathbf{V}=k\left[\left(\sin \theta_{1}-\sin \theta_{2}\right) \mathbf{X}_{0}-\left(\cos \theta_{1}+\cos \theta_{2}\right) \mathbf{Z}_{0}\right]$.

Since $E_{2}$ is given by the following equation :

$$
E_{2}=\frac{i k \mathrm{e}^{i k R_{0}}}{4 \pi R_{0}} \int_{-X}^{X} \int_{-Y}^{Y}\left(a \zeta_{x}^{\prime}+c \zeta_{y}^{\prime}-b\right) \mathrm{e}^{i \mathbf{v} \cdot \mathbf{r}} \mathrm{d} x \mathrm{~d} y
$$

and since we could write a similar formula for the reference field $E_{20}$, as the one given by equation (B.6), but for a perfectly conducting surface with a horizontally polarized incident wave :

$$
E_{20}=\frac{2 i k \mathrm{e}^{i k R_{0}} X Y \cos \theta_{1}}{\pi R_{0}} \text { with } \rho=\frac{E_{2}}{E_{20}} .
$$

Therefore equation (1) (Sect. 3.2) should be written as :

$$
\begin{aligned}
\rho= & \frac{1}{8 X Y \cos \theta_{1}} \times \\
& \times \int_{-X}^{X} \int_{-Y}^{Y}\left(a \zeta_{X}^{\prime}+c \zeta_{y}^{\prime}-b\right) \mathrm{e}^{i \mathbf{v} \cdot \mathbf{r}} \mathrm{d} x \mathrm{~d} y .
\end{aligned}
$$

Consequently, equation (9) (Sect. 3.2) should be corrected by replacing 44 by 8 » appeared in the factor in front of the integral, assigned by (A.6)

$\rho$ for a one dimensionally rough surface of finite conductivity is given by equation (44) (Sect. 3.2). Therefore $\langle\rho\rangle$ of equation (5) (Sect. 5.2) should be written as :

$$
\langle\rho\rangle=F_{2} \chi\left(v_{z}\right) \rho_{0}
$$

with

$$
\begin{gathered}
F_{2}=\sec \theta_{1}\left\{\frac{1+\cos \left(\theta_{1}+\theta_{2}\right)}{\cos \theta_{1}+\cos \theta_{2}}\right\} \\
\chi\left(v_{z}\right)=\left\langle\mathrm{e}^{i v_{z} \zeta}\right\rangle \\
\rho_{0}=\operatorname{sinc} v_{x} L
\end{gathered}
$$

Equation (16) (Sect. 5.2) should be expressed as :

$$
\begin{aligned}
\left\langle\mathrm{e}^{i v_{z}\left(\zeta_{1}-\zeta_{2}\right)}\right\rangle & =\int_{-\infty}^{\infty} \int_{-\infty}^{\infty} W\left(z_{1}, z_{2}\right) \mathrm{e}^{i v_{z}\left(z_{1}-z_{2}\right)} \mathrm{d} z_{1} \mathrm{~d} z_{2} \\
& =\chi_{2}\left(v_{z},-v_{z}\right) .
\end{aligned}
$$

The factor in front of the integrals, in both equations (22) and (24) (Sect. 5.2) should be :

$$
\frac{F_{2}^{2}}{4 L^{2}}
$$

Equation (25) (Sect. 5.2) should be written as :

$$
\langle\rho\rangle=F_{3} \chi\left(v_{z}\right) \rho_{0}
$$

with $A=4 X Y$, to replace $A=X Y$ (given consequently after Eq. (25)) with

$$
F_{3}=\frac{1+\cos \theta_{1} \cos \theta_{2}-\sin \theta_{1} \sin \theta_{2} \cos \theta_{3}}{\cos \theta_{1}\left(\cos \theta_{1}+\cos \theta_{2}\right)} .
$$

Following the correction of equation (25) (Sect. 5.2) as given above, then we should correct both equation (5) (Sect. 5.3) and equation (6) (Sect. 5.3) as follows :

$$
\begin{aligned}
& \langle\rho\rangle=F_{3} \rho_{0} \exp \left[\frac{-2 \pi^{2} \sigma^{2}}{\lambda^{2}}\left(\cos \theta_{1}+\cos \theta_{2}\right)^{2}\right] \\
& \langle\rho\rangle_{\text {spec }}=F_{3} \exp \left[-\frac{1}{2}\left(\frac{4 \pi \sigma \cos \theta_{1}}{\lambda}\right)^{2}\right] .
\end{aligned}
$$

Equation (10) (Sect. 5.3) should be given as :

$$
\sqrt{g}=\left|v_{z} \sigma\right|=\frac{2 \pi \sigma}{\lambda}\left(\cos \theta_{1}+\cos \theta_{2}\right) \text {. }
$$

The following is the corrected expression for $D\{\rho\}$, given in section 5.3, consequently after equation (10) :

$$
\begin{aligned}
D\{\rho\}=-\frac{F_{2}^{2}}{2 L^{2}} \int_{-\infty}^{\infty} & \mathrm{e}^{i v_{x} \tau-g} \times \\
& \times \sum_{m=1}^{\infty} \frac{g^{m}}{m !} \mathrm{e}^{-m \tau^{2} / \mathrm{T}^{2}} \mathrm{~d} \tau .
\end{aligned}
$$

Equation (52) (Sect. 5.3) should be written as :

$$
\langle\rho\rangle\langle\rho\rangle^{*}= \begin{cases}\mathrm{e}^{-g \rho_{0}^{2}} \text { for } v_{x y}=0 \\ 0 & \text { for } v_{x y} \neq 0 .\end{cases}
$$

Equation (53) (Sect. 5.3) should be expressed as :

$$
\left\langle\rho \rho^{*}\right\rangle=D\{\rho\}+\mathrm{e}^{-g \rho_{0}^{2}} .
$$

The symbol letter $F$ appears in section 5.3, equation numbers ((13)-(66)), should be replaced by $F_{2}$ (one dimensional roughness), and $F_{3}$ (two dimensional roughness).

Equation (63) (Sect. 5.3) should be written as :

$$
\left\langle\left|R_{1}\right|^{2}\right\rangle=\frac{A}{\pi r^{2}} \frac{v_{x y}^{4}}{v_{z}^{4}} \cot ^{2} \beta_{0} \exp \left(\frac{-\tan ^{2} \beta}{\tan ^{2} \beta_{0}}\right) \text {. }
$$

Equation (3) (Sect. 5.4) should be given as :

$$
\begin{array}{r}
\langle\rho\rangle=\frac{-\left[(1+\langle R\rangle) \cos \theta_{2}-(1-\langle R\rangle) \cos \theta_{1}\right]}{2 \cos \theta_{1}} \times \\
\quad \times \operatorname{sinc} v_{x} L_{*} \chi\left(v_{z}\right) \quad(\mathrm{A} .18)
\end{array}
$$


The phase difference between two waves 1 and 2 scattered at the points $\zeta\left(x_{1}\right)$ and $\zeta\left(x_{2}\right)$ of the surface is :

$$
\Delta \phi=\mathbf{V} \cdot\left(\mathbf{r}_{1}-\mathbf{r}_{2}\right)
$$

with $\mathbf{V}$ given by $\left(\theta_{3}=0\right)$ :

$$
\begin{gathered}
\mathbf{V}=k\left[\left(\sin \theta_{1}-\sin \theta_{2}\right) \mathbf{X}_{0}-\left(\cos \theta_{1}+\cos \theta_{2}\right) \mathbf{Z}_{0}\right] \\
\mathbf{r}_{1}=x_{1} \mathbf{X}_{0}+\zeta_{1}\left(x_{1}\right) \mathbf{Z}_{0} \\
\mathbf{r}_{2}=x_{2} \mathbf{X}_{0}+\zeta_{2}\left(x_{2}\right) \mathbf{Z}_{0} \\
\left(\mathbf{r}_{1}-\mathbf{r}_{2}\right)=\left(x_{1}-x_{2}\right) \mathbf{X}_{0}+\left(\zeta_{1}-\zeta_{2}\right) \mathbf{Z}_{0} .
\end{gathered}
$$

Therefore equation (8) (Sect. 7.3) should be written as :

$$
\begin{aligned}
\Delta \phi_{12}=\frac{2 \pi}{\lambda} & {\left[\left(x_{1}-x_{2}\right)\left(\sin \theta_{1}-\sin \theta_{2}\right)-\right.} \\
& \left.-\left(\zeta_{1}-\zeta_{2}\right)\left(\cos \theta_{1}+\cos \theta_{2}\right)\right]
\end{aligned}
$$

\section{Appendix B.}

The definition of the reference field ; expression in 2$D$ of the field $E_{20}$ for a surface having a finite conductivity.

$E_{20}$ is the field reflected in the direction of specular reflection $\left(\theta_{1}=\theta_{2}\right)$ by a smooth, finite conductive plane with the same dimensions under the same angle of incidence at the same distance, when the incident wave is vertically polarized. When $\theta_{1}=\theta_{2}$; $\zeta=\zeta^{\prime}=0$.

From equation (5) is derived :

$$
a=2 \sin \theta_{1}=2 \sin \theta_{2}
$$

from equation (6)

$$
b=2 R \cos \theta_{1}
$$

and from equation (2)

$$
\begin{aligned}
& v_{x}=0 \\
& v_{z}=-2 k \cos \theta_{1} .
\end{aligned}
$$

So the reflected field $E_{20}$ is written :

$E_{2}=E_{20}=\frac{i k \mathrm{e}^{i k R_{0}}}{4 \pi R_{0}} \int_{-x}^{x} \int_{-y}^{y}-2 R \cos \theta_{1} \mathrm{~d} x \mathrm{~d} y$

$E_{20}=\frac{-2 i k \mathrm{e}^{i k R_{0}} R \cos \theta_{1} X Y}{\pi R_{0}}$

with $0 \leqslant R<1$. For a vertically polarized incident wave.

$R_{0}$ is the distance of the observation point from the origin [1].

\section{References}

[1] Beckmann P. and Spizzichino A., The scattering of electromagnetic waves from rough surfaces (The Mac Millan Company, New York) 1963.

[2] Beckmann P., The reflection of electromagnetic waves and synthesis of media. Acta Tech. CSAV1 (1956) pp. 251-267.
[3] Galal T., Awajan A. and Mignot J., The distribution of EM waves scattering over cutaneous surfaces. Presented at IEEE/Eight Annual Conference of the Engineering in Medicine and Biology Society, Forth Worth (Texas) Nov. 710, 1986, pp. 1410-1413. 